\title{
Cooperative Localisation of UAVs in a GPS-denied Environment Using Bearing Measurements
}

\author{
Lvtianyang Zhang ${ }^{1}$, Mengbin Ye ${ }^{1}$, Brian D.O. Anderson ${ }^{1,2}$, Peter Sarunic ${ }^{3}$ and Hatem Hmam ${ }^{3}$
}

\begin{abstract}
This paper studies the problem of localising a Global Positioning System (GPS)-denied Unmanned Aerial Vehicle (UAV) in two-dimensional space. Suppose there are two vehicles, one which is equipped with GPS and the other is GPSdenied (but has an inertial navigation system (INS) and so is able to determine its trajectory in a local coordinate frame, but not a global coordinate frame). The GPS-equipped vehicle broadcasts its global coordinates to the GPS-denied vehicle and the GPS-denied vehicle also obtains, in its local coordinate frame, a bearing measurement of the GPS-equipped UAV. The paper shows that with four or more such measurements and generic trajectories of the two UAVs, localisation in a global coordinate frame of the GPS-denied UAV is achievable. Certain nongeneric trajectories for which localisation is impossible are also identified. While in the first instance, the solution assumes zero noise in the measurements, the techniques are then extended to deal with the presence of measurement noise.
\end{abstract}

\section{INTRODUCTION}

UAVs which are being used for surveillance will frequently operate in a formation, and to maintain the formation shape, it will generally be necessary for one or more of the UAVs to be able to localise others in the formation [1]. There are however two constraints which may make this task difficult. First, it may be that there is GPS denial (through jamming by an enemy, or lack of line of sight) which may affect a number of the UAVs. Second, given that in most cases the primary objective of the formation of UAVs is to sense or otherwise study something outside of the formation, there is pressure to minimize on board sensors associated with inter-vehicle localisation. These constraints motivate the problem we study in this paper, which is to explain how a GPS-denied vehicle, henceforth called Agent B, can be localised by limited measurements from a GPSequipped vehicle, called Agent A henceforth.

Although this paper's approach to cooperative localisation is primarily focused on application to UAVs, the derived formulations and proposed solutions are equally applicable to any autonomous vehicle operating cooperatively in a

\footnotetext{
${ }^{1}$ Lvtianyang Zhang, Mengbin Ye and Brian D.O. Anderson are with the Research School of Engineering, Australian National University. ${ }^{2}$ Brian D.O. Anderson is also with Hangzhou Dianzi University, Hangzhou, China and Data61-CSIRO (formerly NICTA Ltd.) in Canberra, A.C.T., Australia. ${ }^{3}$ Peter Sarunic and Hatem Hmam are with the Australian Defence Science and Technology Group (DSTG), Adelaide, Australia. This work was supported in part by Data61-CSIRO (formerly NICTA) and the Australian Research Council (ARC) under DP-130103610 and DP-160104500. The authors would also like to thank Mark Knight from Defence Science and Technology Group for his suggestions to enhance the paper.

\{u5870605, Mengbin.Ye, Brian.Anderson\}eanu.edu.au \{Peter.Sarunic, Hatem.Hmam\}@dsto.defence.gov.au
}

two-dimensional space and capable of taking bearing measurements. These include AUVs (autonomous underwater vehicles), marine surface vessels or ground vehicles. Indeed, the vehicles need not be autonomous.

In the context of cooperative UAVs, a reasonable scenario for the two-dimensional problem is when UAVs are travelling approximately horizontally or the elevation angle of Agent A relative to Agent B is small.

There has been limited work on related problems, although many inter-vehicle localisation problems start with an assumption that any inter-agent measurements will include range and bearing, or equivalently relative position. Exceptions do however exist and we outline some.

In [2], a method of controlling a three agent triangular formation by using bearing-only measurements in a local coordinate system is studied. An approach uses bearings measured by a moving observer combined with knowledge of the observer's motion to localise a stationary target [3]. A fixed target localisation problem using passive bearing measurements is studied using total least squares in [4]. The work of [5] studies bearing-only measurement localisation of a fixed target by a moving observer. Noise corrupted measurements and uncertain observer position are handled using maximum likelihood estimation. In a more ambitious study [6], bearing-only measurements are used by one agent to localise a second agent in an ambient two-dimensional space given that both agents are restricted to moving in circular orbits and with no inter-agent communication. With the replacement of bearing-only sensing by range-only sensing, the same problem is treated in [7]. In contrast, [8] estimates the trajectory of a target with constant velocity, for an arbitrary observer trajectory using noisy sensor data.

Utilising inter-agent communication, an agent formation is localised in [9] using bearing-only measurements apart from a further single range measurement. The paper [10] localises a stationary network using noisy bearing-only measurements; exact positions of at least two anchor agents in the global map are required. In contrast to these studies which either place restrictions on the trajectories or require additional information, in this paper, we will focus on localisation of freely moving agents using discrete bearing measurements. We do however permit Agent A to communicate its position information to Agent B (but not vice versa).

We assume in this paper that Agent B has inertial navigation capability (INS), which means that it can construct a map of where it is in its own local coordinate system. Further, it can receive information transmitted at discrete time instants from Agent A, describing Agent A's global position at the 
same transmission times. We assume also that Agent B can determine, in its local coordinate basis, the direction from which the received transmissions arrive, (e.g. Agent B uses an antenna array to receive radio frequency (RF) signals sent by Agent A). Agent B must localise itself in the global coordinate basis by putting together its knowledge of its own trajectory, with knowledge of Agent A's global positions at discrete instants of time, together with the bearings to Agent A. The cooperative aspect of this work is in the fact that Agent B is able to obtain Agent A's global positions. For simplicity, this paper focuses on two UAVs rather than a group of UAVs but the problem can obviously be generalized by increasing the assumed number of GPS-equipped and/or GPS-denied vehicles.

While, in the real world, there is always measurement noise to be reckoned with, we study initially the noiseless case. We explain how the localisation task is really one of determining the location of the origin and the orientation of the GPS-denied agent's local coordinate frame, these being defined by three scalar variables. However, it turns out that if we take three bearing measurements, the localisation problem can only ever be solved up to a binary ambiguity for generic situations. In the absence of further a priori information on the position of the GPS-denied agent, a fourth measurement is required to disambiguate the pair of solutions. In the noiseless case, the localisation solution can be obtained using linear equations alone. There are special trajectories for which the technique proposed in this paper fails and these are identified in the paper.

We turn then to a consideration of measurement noise. We propose a two step approach in solving the localisation problem for noisy measurements:

1) Solve a Generalised Trust Region Subproblem (GTRS) to obtain a solution which minimises a quadratic performance index subject to a quadratic constraint. This quadratic constraint ensures that the solution obeys the requirement that a certain matrix be orthogonal.

2) Use the information available at this point as the initial condition of a gradient descent maximum likelihood estimation problem in which the parameters correspond to the origin and axis orientation of the local coordinate basis relative to the global coordinate basis.

The paper is structured as follows. Following a formal problem definition in the next section, we discuss approaches to solving the noiseless problem in Section III, which also covers the issue of the required number of measurements, and the nature of the special trajectories for which solutions are not possible. Section IV shows how to cope with noisy measurements, through use of a gradient-based maximum likelihood estimate procedure, initialized using a GTRS algorithm. Section V contains concluding remarks, and directions for future work.

\section{Problem Formulation AND BACKGRound}

We consider two sensing agents which are moving in twodimensional space, i.e. the UAVs are flying in a plane parallel to the Earth's surface. Let Agent A be GPS-equipped, while the other agent, call it Agent B, has INS but is GPS-denied. Agent A broadcasts its position (in global coordinates) at discrete time instants, indexed as $k=1,2, \ldots$, and this broadcast is received by Agent B. This also allows Agent $B$ to sense the direction from which the signal arrived, i.e. the bearing of Agent A in the local coordinates of Agent B.

The Euclidean norm of a vector is $\|\cdot\|$. For the $k^{t h}$ time instant, we denote the following. The global coordinates of Agent $\mathrm{A}$ are $\boldsymbol{P}_{A k} \in \mathbb{R}^{2 \times 1}$, Agent A's local coordinates (i.e. its coordinates in the coordinate frame associated with Agent B) are denoted as $\boldsymbol{P}_{A k}^{\prime} \in \mathbb{R}^{2 \times 1}$, Agent B's global coordinates as $\boldsymbol{P}_{B k} \in \mathbb{R}^{2 \times 1}$ and Agent B's local coordinates as $\boldsymbol{P}_{B k}^{\prime} \in \mathbb{R}^{2 \times 1}$. In these coordinate vectors, in their respective frames, the first entry refers to east, and the second entry to north. Coordinates expressed in vector form can be transferred from one coordinate frame to another coordinate frame through a rotation matrix $R \in \mathbf{S O}(2)$ (i.e. $R$ is a orthogonal matrix with $\operatorname{det}(R)=1$ ) and a translation vector $T \in \mathbb{R}^{2 \times 1}$ and vice versa. It follows that

$$
\begin{gathered}
\boldsymbol{P}_{A k}^{\prime}=R \boldsymbol{P}_{A k}+T \\
\boldsymbol{P}_{B k}=R^{-1}\left(\boldsymbol{P}_{B k}^{\prime}-T\right)
\end{gathered}
$$

We assume that the overall measurement time interval is sufficiently short such that the rotation matrix and translation vector are time invariant. Equivalently, the INS drift is negligible during the overall measurement interval. Consider Fig. 1, which captures the UAV positions at the $k^{\text {th }}$ time instant, in Agent B's local coordinate basis. We define $\theta_{k}$ as the bearing angle between a line parallel to the axis $Y^{\prime}$, which starts from Agent $\mathrm{B}$ and extends in the positive $Y^{\prime}$ direction, and the line between Agent B and Agent A. This value $\theta_{k}$ is the bearing of Agent $A$ measured in the local coordinate basis of Agent B, which is available to Agent B as mentioned at the beginning of this section.

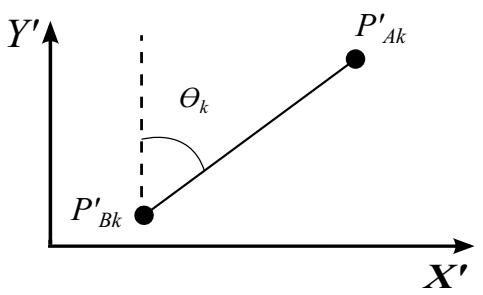

Fig. 1. Two agents as viewed in Agent B's local coordinate.

$$
\begin{aligned}
& \text { Denote } \boldsymbol{P}_{A k}=\left[u_{k}, v_{k}\right]^{\top}, \boldsymbol{P}_{B k}^{\prime}=\left[x_{k}^{\prime}, y_{k}^{\prime}\right]^{\top}, T=\left[t_{1}, t_{2}\right]^{\top} \\
& \text { and } \\
& \qquad R=\left[\begin{array}{cc}
\cos \alpha & \sin \alpha \\
-\sin \alpha & \cos \alpha
\end{array}\right]=\left[\begin{array}{cc}
m & n \\
-n & m
\end{array}\right]
\end{aligned}
$$

The rotation matrix $R$ in equation (3) can be parameterised by the rotation matrix angle $\alpha$, but for the material below it proves more convenient to use the separate parameters $m, n$ and note that $m^{2}+n^{2}=1$. From Figure 1 and our definitions of $\boldsymbol{P}_{A k}, \boldsymbol{P}_{B k}^{\prime}, R, T$, it follows that

$$
\begin{aligned}
& \sin \theta_{k}=\left(m u_{k}+n v_{k}+t_{1}-x_{k}^{\prime}\right) / z \\
& \cos \theta_{k}=\left(-n u_{k}+m v_{k}+t_{2}-y_{k}^{\prime}\right) / z
\end{aligned}
$$


where $z=\left\|R \boldsymbol{P}_{A k}+T-\boldsymbol{P}_{B k}^{\prime}\right\|$. Combing the above yields $\cos \theta_{k}\left(m u_{k}+n v_{k}+t_{1}-x_{k}^{\prime}\right)=\sin \theta_{k}\left(-n u_{k}+m v_{k}+t_{2}-y_{k}^{\prime}\right)$

In (5), we observe that there are four unknown variables $m$, $n, t_{1}$ and $t_{2}$. Combining the constraints from the orthogonal matrix in (3), we have a total of three independent variables. Therefore, we expect that any localisation solution would require at least three bearing measurements.

\section{Localis ation With Noiseless Measurements}

In this section, we consider noiseless bearing measurements. Generically, the minimum number of measurements required to obtain the unique and correct localisation solution is four. Some special cases are also investigated; at best, a partial localisation solution is obtainable for these cases.

For an arbitrary matrix, A, we denote the $i^{t h}$ row as $A(i$,$) , the j^{t h}$ column as $A(, j)$. Every measurement $\theta_{k}$ imposes a linear constraint on the unknowns $m, n, t_{1}, t_{2}$, which is observed from equation (5). Suppose we take $K$ measurements. Then the $K$ linear constraints can be expressed as a system of linear equations which has the following form

$$
\left[\begin{array}{cccc}
a_{1,1} & a_{1,2} & a_{1,3} & a_{1,4} \\
\vdots & \vdots & \vdots & \vdots \\
a_{K, 1} & a_{K, 2} & a_{K, 3} & a_{K, 4}
\end{array}\right]\left[\begin{array}{c}
t_{1} \\
t_{2} \\
m \\
n
\end{array}\right]=\left[\begin{array}{c}
b_{1} \\
\vdots \\
b_{K}
\end{array}\right]
$$

This equation can be expressed as

$$
A_{K} x=B_{K}
$$

where $x$ is the vector of unknown variables. The elements of $A_{K}$ are as follows

$$
\begin{aligned}
& \text { - } a_{k, 1}=-\cos \theta_{k} \\
& \text { - } a_{k, 2}=\sin \theta_{k} \\
& \text { - } a_{k, 3}=v_{k} \sin \theta_{k}-u_{k} \cos \theta_{k} \\
& \text { - } a_{k, 4}=-\left(u_{k} \sin \theta_{k}+v_{k} \cos \theta_{k}\right) \\
& \text { - } b_{k}=-y_{k}^{\prime} \sin \theta_{k}-x_{k}^{\prime} \cos \theta_{k}
\end{aligned}
$$

Theorem 1. Suppose that three bearing measurements, $\theta_{1}, \theta_{2}, \theta_{3}$, are obtained by Agent $B$ (in other words $K=$ 3). Using these measurements and the corresponding three global coordinates of Agent A and three local coordinates of Agent B, then there is either a unique localisation solution or two localisation solutions. If there are two solutions then it is impossible to disambiguate the solutions without further data.

Proof. From the three bearings and the positions of agents A (respectively Agent B) in the global coordinate frame (respectively local coordinate frame), and recalling that the rotation matrix is orthogonal, it follows that

$$
\begin{gathered}
A_{3} x=B_{3} \\
m^{2}+n^{2}=1
\end{gathered}
$$

Suppose the matrix $A_{3}$ in equation (6) has rank three. While this is generically true, in the sequel, we also explore when the matrix $A_{K}$ is not of full rank. From the set of linear equations (8), we can generically eliminate $t_{1}$ and $t_{2}$ to obtain

$$
a n+b m=c
$$

where $a, b$ and $c$ are known constants. Equations (9) and (10) represent a circle and a straight line in the variables $m$ and $n$. A straight line intersects a circle either a) never, b) at a unique point or c) at two points. The type of intersection that occurs (and hence the localisation solution) can be described by a value $d$ where $d=a^{2}+b^{2}-c^{2}$. If $d>0$, we have two real localisation solutions or in other words, there are two $R$ matrices and two $T$ vectors which satisfy (8) and (9). Consequently, we obtain two sets of possible coordinates of Agent B in the global map, which can be obtained by expression (2). Call the two sets of positions $\mathcal{G}=\left\{\boldsymbol{P}_{B 1}, \boldsymbol{P}_{B 2}, \boldsymbol{P}_{B 3}\right\}$ and $\mathcal{G}^{*}=\left\{\boldsymbol{P}_{B 1}{ }^{*}, \boldsymbol{P}_{B 2}{ }^{*}, \boldsymbol{P}_{B 3}{ }^{*}\right\}$. One can also view this as Agent B obtaining two possible sets of coordinates of Agent $A$ in the local map, given as $\mathcal{H}=\left\{\boldsymbol{P}_{A 1}^{\prime}, \boldsymbol{P}_{A 2}^{\prime}, \boldsymbol{P}_{A 3}^{\prime}\right\}$ and $\mathcal{H}^{*}=\left\{\boldsymbol{P}_{A 1}^{\prime}{ }^{*}, \boldsymbol{P}_{A 2}^{\prime}{ }^{*}, \boldsymbol{P}_{A 3}^{\prime}{ }^{*}\right\}$.

If $d=0$, we have a unique localisation solution (a unique $R$ and unique $T$ ). The case $d=0$ is nongeneric as discussed further in Remark 1 below. If $d<0$ then no real solutions exist. However, note that values of $A_{3}$ and $B_{3}$ are obtained from a real flight scenario. Combining this with the fact that the rotation matrix and translation vector are timeinvariant over the total measurement time interval, one can then conclude that $d$ will always be nonnegative. It follows that there must exist at least one real localisation solution, but that for $d>0$ it is impossible to disambiguate the two solutions without further information.

We next show that with a fourth bearing measurement, one can obtain a unique localisation solution in generic scenarios. In the sequel, we also investigate some special cases where only partial localisation is achievable, even with four bearing measurements.

Theorem 2. Suppose that four bearing measurements are obtained by Agent $B$ given as $\theta_{1}, \ldots, \theta_{4}$ (i.e. $K=4$ ). Using these measurements, and the corresponding set of four global coordinates of Agent $A$ and four local coordinates of Agent B, then there is a unique localisation solution for generic flight paths of Agent A and Agent B.

Proof. A unique localisation solution is obtained if there is a unique $x$ which satisfies

$$
A_{4} x=B_{4}
$$

and this occurs if $\operatorname{det}\left(A_{4}\right) \neq 0$. Notice that $\theta_{k}$ is a continuous function of $u_{k}, v_{k}, x_{k}^{\prime}, y_{k}^{\prime}$ and observe that the determinant of a matrix is a continuous function of the elements of that matrix. Suppose that $\operatorname{det}\left(A_{4}\right)=0$. Then slight perturbations to a value, say $u_{k}$, will result in $\operatorname{det}\left(A_{4}\right) \neq 0$. One can see that only specific algebraic combinations of $u_{k}, v_{k}, x_{k}^{\prime}, y_{k}^{\prime}$ will yield $\operatorname{det}\left(A_{4}\right)=0$; these are explored in detail later. Generically however, one has $\operatorname{det}\left(A_{4}\right) \neq 0$ and thus a unique localisation solution is obtained. 


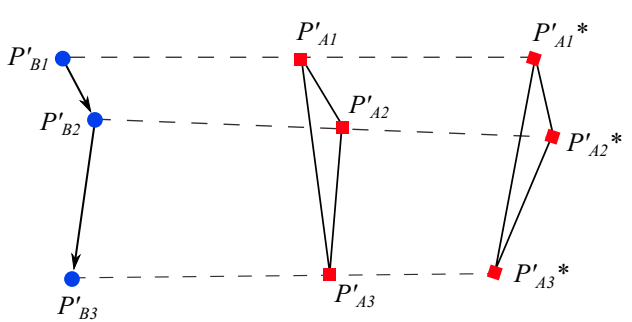

Fig. 2. Example scenario where three measurements gives two solutions, as viewed from the local coordinates of Agent B.

\section{A. Geometric Explanation for Theorems 1 and 2}

Using an example, we provide an explanation of Theorems 1 and 2, and in particular, how two solutions arise when using three bearing measurements and a fourth measurement disambiguates the solutions. Consider Fig. 2, which is in Agent B's local coordinate frame (one can also create the same diagram in the global coordinate frame). The dashed lines represent the bearing measurement to Agent A, as measured by Agent $\mathrm{B}$. Let the triangle formed by the three points of $\mathcal{H}$ (respectively $\mathcal{H}^{*}$ ) be $\Delta_{\mathcal{H}}$ (respectively $\Delta_{\mathcal{H}^{*}}$ ). Notice that $\Delta_{\mathcal{H}}$ and $\Delta_{\mathcal{H}^{*}}$ are of the same size and shape; they differ by a rotation and a translation (but not a reflection). Both $\Delta_{\mathcal{H}}$ and $\Delta_{\mathcal{H}^{*}}$ satisfy (8) and (9) and cannot be disambiguated; this is the geometric explanation of Theorem 1 .

Now consider when four bearing measurements are taken by Agent B. It is straightforward to observe that, generically, a fourth measurement disambiguates the two solutions to yield the correct, unique localisation solution. Suppose that the flight path of Agent B is such that $\boldsymbol{P}_{B 4}^{\prime}$ lies on the line connecting $\boldsymbol{P}_{A 4}^{\prime}$ and $\boldsymbol{P}_{A 4}^{\prime}{ }^{*}$ and does not lie between $\boldsymbol{P}_{A 4}^{\prime}$ and $\boldsymbol{P}_{A 4}^{\prime}{ }^{*}$. Then, as shown in Fig. 3 where the line is labelled $l_{4}$, there are two solutions which satisfy equation (11). In this nongeneric scenario, $\operatorname{rank}\left(A_{4}\right)<4$ and we have two solutions which cannot be disambiguated. However, if $\boldsymbol{P}_{B 4}^{\prime}$ is anywhere else, we obtain a unique solution. Note that if $\boldsymbol{P}_{B 4}^{\prime}$ lies on $l_{4}$ but is between $\boldsymbol{P}_{A 4}^{\prime}$ and $\boldsymbol{P}_{A 4}^{\prime}{ }^{*}$, a unique solution is obtained because we also have directional information from $\theta_{4}$ (relative to Agent B's North).

\section{B. Special Cases}

One special case has already been explored above and we now explore other nongeneric flight scenarios which result in $\operatorname{rank}\left(A_{4}\right)<4$ (and thus a unique localisation solution is not obtainable). Note that when we discuss flight paths of the UAVs, e.g. Agent A, we refer to the straight line connecting the point $\boldsymbol{P}_{A k}$ to $\boldsymbol{P}_{A(k+1)}$ as opposed to the physical motion of the UAV from $\boldsymbol{P}_{A k}$ to $\boldsymbol{P}_{A(k+1)}$ (which may include smooth curved flight). Remark 3 discusses special cases for $K>4$ bearing measurements.

1) The four measured bearings are equal in value: Consider a flight scenario of Agents A and B such that $\theta_{1}=\theta_{2}=\theta_{3}=\theta_{4}$. If this occurs then the line joining $\boldsymbol{P}_{A k}$ to $\boldsymbol{P}_{B k}$ is parallel to the line joining $\boldsymbol{P}_{A(k+1)}$ to $\boldsymbol{P}_{B(k+1)}$, for all $k<K-1$ (this parallel line property also holds true when viewed from the local coordinate of Agent B). It is

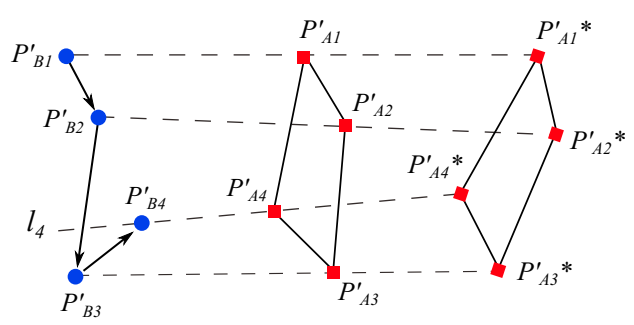

Fig. 3. Example scenario (nongeneric) where four measurements gives two solutions: $\boldsymbol{P}_{B 4}^{\prime}$ must lie on the line $l_{4}$.

straightforward to verify that the columns $A(, 1)$ and $A(, 2)$ are linearly dependent and thus $\operatorname{rank}\left(A_{4}\right)<4$. Suppose that the submatrix formed by columns $A_{4}(, 2), A_{4}(, 3)$ and $A_{4}(, 4)$, call it $\widetilde{A}$, is full rank (immediately below, we explore the case where it is not full rank). From expression (6) and the fact that the first two columns of $A_{4}$ are linearly dependent but the last three columns are linearly independent, it is straightforward to verify that $\operatorname{rank}\left(A_{4}\right)=3$ and we can obtain a unique value for $m$ and $n$ satisfying $m^{2}+n^{2}=1$. However, $t_{1}=\rho t_{2}$ where $\rho \in \mathbb{R}$, i.e. the translation vector $T$ is determined only up to scaling.

We illustrate this with an example. The flight paths are shown in Fig. 4, as viewed from Agent B's local coordinate frame (one could also view this from the global coordinate frame). Suppose that the true positions of Agent A are $\left\{\boldsymbol{P}_{A 1}^{\prime}, \boldsymbol{P}_{A 2}^{\prime}, \boldsymbol{P}_{A 3}^{\prime}, \boldsymbol{P}_{A 4}^{\prime}\right\}$. However, one could slide the position set along the lines representing the bearing measurement, e.g. $\left\{\boldsymbol{P}_{A 1}^{\prime}{ }^{*}, \boldsymbol{P}_{A 2}^{\prime}{ }^{*}, \boldsymbol{P}_{A 3}^{\prime}{ }^{*}, \boldsymbol{P}_{A 4}^{\prime}{ }^{*}\right\}$, and equation (11) continues to hold. Thus, one obtains a unique rotation matrix $R$ but the translation vector is determined up to scaling. Note that this special case is of interest to certain formation control tasks as discussed further in Remark 2 below.

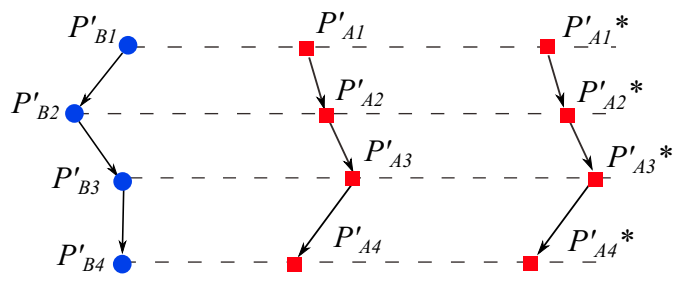

Fig. 4. Example scenario of when the four bearing measurements are equal. The dashed lines represent the bearing measurements.

2) The four measured bearings are the same and the flight path of Agent $A$ is a straight line: Suppose that in addition to having $\theta_{1}=\theta_{2}=\theta_{3}=\theta_{4}$, Agent A flies in a way such that the points $\left\{\boldsymbol{P}_{A 1}, \boldsymbol{P}_{A 2}, \boldsymbol{P}_{A 3}, \boldsymbol{P}_{A 4}\right\}$ are collinear. This can be expressed as $v_{k}=\lambda u_{k}+\varphi, \forall k$, where $\lambda, \varphi \in \mathbb{R}$ are constants. It is straightforward to verify that the matrix $\widetilde{A}$ defined in the section above is not full rank and in fact $\operatorname{rank}\left(A_{4}\right)=2$. In addition to determining $T$ up to scaling, there are two solutions for $m, n$ satisfying $m^{2}+n^{2}=1$. This implies that there are two solutions for $R$ and they cannot be disambiguated. However, note that if the flight path of Agent A is a straight line (i.e. $v_{k}=\lambda u_{k}+\varphi, \forall k$ ) but the four 
bearings are not the same then there is a unique localisation solution, i.e. $\operatorname{rank}\left(A_{4}\right)=4$.

3) Agent A is stationary: If Agent A is stationary we have, from the view of Agent $\mathrm{B}$, a problem similar to the standard problem involving localisation of a stationary target. With $u_{1}=\ldots=u_{4}$ and $v_{1}=\ldots=v_{4}$, we compute that $\operatorname{rank}\left(A_{4}\right)=2$. However, unlike the above scenario, here we are able to obtain a unique translation vector $T$ but we do not have a unique $R$ matrix. Specifically, the system of equations (11) is satisfied by any pair $m, n$ such that $m^{2}+n^{2}=1$.

In the extreme case, the points $\boldsymbol{P}_{B k}, \forall k$ are collinear with the stationary point $\boldsymbol{P}_{A}$. This yields $\operatorname{rank}\left(A_{4}\right)=1$ and one obtains $T$ up to scaling and a nonunique $R$.

\section{Simulation}

A simulation is provided to show the conclusions obtained by Theorems 1 and 2. More extensive simulations appear in the following section when we study noisy bearing measurements. Table I provides the data used by Agent B to calculate the localisation solution. Using the first three sets of information from Table I, Agent B obtains two solutions which are detailed in Table II. Using a fourth set of information, Agent B obtains the correct unique solution (Solution One of Table II). The simulations reflect the statements made in Theorems 1 and 2 .

Remark 1 (The case where $d=0$ ). It is possible to analytically obtain expressions for $\theta_{1}, \ldots, \theta_{3}, \boldsymbol{P}_{A 1}, \ldots, \boldsymbol{P}_{A 3}$ and $\boldsymbol{P}_{B 1}^{\prime}, \ldots, \boldsymbol{P}_{B 3}^{\prime}$ such that $d=0$, where $d$ is defined below (10). However, these will correspond to nongeneric combinations of $\boldsymbol{P}_{A k}$ and $\boldsymbol{P}_{B k}^{\prime}$. In other words, only specific fight patterns for both Agent $A$ and Agent $B$ will yield $d=0$. We therefore focus on $d>0$ and using a fourth measurement to disambiguate the solutions.

Remark 2 (Implications of four equal bearing measurements). The conclusion drawn in Section III-B.1 is of importance when considering formation control of UAVs, for which a common objective is to move as a formation; this requires velocity consensus, see e.g. [11]. Notice that, for $\theta_{1}=\ldots=\theta_{4}$, Agent $A$ and Agent $B$ have velocity consensus in the direction perpendicular to the line representing the bearing angle, $\theta_{k}$. See Fig. 4 for example. This implies that the proposed localisation method is not suitable for formation control involving velocity consensus of GPS-denied UAVs. Modifications may be required, or this method may be combined with extra information obtained separately.

\section{Noisy Bearing Measurements}

In Theorem 2, we conclude that four measurements yield a unique solution in the absence of noise. However, in the noisy case, the algorithm leads to a solution which is inconsistent because in general (9) does not hold. This is true even if $K>4$ measurements are used to obtain a linear least squares solution. In this section, we provide a two-stage approach to dealing with the noise. Firstly, we detail a method which solves the localisation problem in the least squares sense, while ensuring that the solution satisfies $m^{2}+n^{2}=1$. In other words, we seek to minimise a quadratic function subject to a single quadratic constraint. Such problems are also known as a Generalised Trust Region Subproblem (GTRS) [12] and while they are typically nonconvex, we show that a unique optimal solution is obtainable. Next, we use the solution to the GTRS as an initialisation point for an iterative gradient descent maximum likelihood estimation algorithm.

Simulations validate the performance of the two step approach. Throughout this section we assume that the bearing angle $\theta_{k}$ is noisy but that the coordinates, $\boldsymbol{P}_{A k}$ and $\boldsymbol{P}_{B k}^{\prime}$, are noise-free. Strictly speaking, bearing noise should be modelled using a von Mises distribution [13]. However, for the levels of noise we are considering, a Gaussian density effectively provides the same performance. Our experience indicates that the standard deviation for bearing noise for most RF based bearing measurements is at most $10^{\circ}$.

\section{A. Generalised Trust Region Subproblem}

For $K \geq 4$, the GTRS is solved by seeking the $x^{*}$ such that

$$
\boldsymbol{x}^{*}=\operatorname{argmin}_{x \in \mathbb{R}^{4}}\left\{\left\|\boldsymbol{A}_{K} \boldsymbol{x}-\boldsymbol{B}_{K}\right\|^{2}: \boldsymbol{x}^{\top} \boldsymbol{D} \boldsymbol{x}=1\right\}
$$

where $\boldsymbol{D}=\operatorname{diag}[0,0,1,1]$. From [12], $x^{*}$ is an optimal solution of (12) if and only if there exists $\lambda \in \mathbb{R}$ such that

$$
\begin{aligned}
\left(\boldsymbol{A}_{K}^{\top} \boldsymbol{A}_{K}+\lambda \boldsymbol{D}\right) \boldsymbol{x} & =\boldsymbol{A}_{K}^{\top} \boldsymbol{B}_{K} \\
\boldsymbol{x}^{\top} \boldsymbol{D} \boldsymbol{x} & =1 \\
\boldsymbol{A}_{K}^{\top} \boldsymbol{A}_{K}+\lambda \boldsymbol{D} & \succ 0
\end{aligned}
$$

and the last equation indicates that the matrix sum on the left is positive definite. The optimal solution is

$$
\boldsymbol{x}^{*}=\left(\boldsymbol{A}_{K}^{\top} \boldsymbol{A}_{K}+\lambda^{*} \boldsymbol{D}\right)^{-1} \boldsymbol{A}_{K}^{\top} \boldsymbol{B}_{K}
$$

where $\lambda^{*}$ is the unique solution of

$$
g(\lambda)=1, \lambda \in \mathcal{I}=\left(-\lambda_{\max }\left(\boldsymbol{D}, \boldsymbol{A}_{K}^{\top} \boldsymbol{A}_{K}\right)^{-1}, \infty\right)
$$

and the function $g(\lambda)=\boldsymbol{y}(\lambda)^{\top} \boldsymbol{D} \boldsymbol{y}(\lambda)$ with

$$
\boldsymbol{y}(\lambda)=\left(\boldsymbol{A}_{K}^{\top} \boldsymbol{A}_{K}+\lambda \boldsymbol{D}\right)^{-1} \boldsymbol{A}_{K}^{\top} \boldsymbol{B}_{K}
$$

Restricting $\lambda$ to the interval $\mathcal{I}$ ensures that (13c) holds. The value

$$
\lambda_{\max }\left(\boldsymbol{D}, \boldsymbol{A}_{K}^{\top} \boldsymbol{A}_{K}\right)=\lambda_{\max }\left(\left(\boldsymbol{A}_{K}^{\top} \boldsymbol{A}_{K}\right)^{-\frac{1}{2}} \boldsymbol{D}\left(\boldsymbol{A}_{K}^{\top} \boldsymbol{A}_{K}\right)^{-\frac{1}{2}}\right)
$$

denotes the smallest generalised eigenvalue of the matrix pair $\left(\boldsymbol{D}, \boldsymbol{A}_{K}^{\top} \boldsymbol{A}\right)$. One can easily check that there is a unique solution to (15), but we leave out the proof due to space limitations.

Remark 3 (Extension to $K>4$ measurements). The special scenarios discussed in Section III-A can be trivially extended to $K>4$ measurements. In a noiseless environment, there is no point in taking further measurements (unless the drift in Agent B's INS is substantial, and Agent B needs to be re-localised). With noise, we take $K \geq 4$ and use the GTRS algorithm. Consider Special Case 1). With no noise, it is straightforward to obtain that $\operatorname{rank}\left(A_{K}\right)<4$ if we have 
$n>K-3$ bearings which are equal, e.g. for $K=10$, if 8 or more of the measured bearings are equal. Although $A_{K}$ may be full rank in the presence of noise, the GTRS algorithm is likely to return an inaccurate localisation estimate. We omit further detailed discussions due to spatial limitations; similar conclusions for the other special cases are easily obtainable.

\section{B. Maximum Likelihood Estimation}

Suppose the measurement of bearings is corrupted by Gaussian noise; i.e. in the $k^{t h}$ measurement, $\widetilde{\theta}_{k}=\theta_{k}+\xi$, where $\xi \sim N\left(0, \sigma^{2}\right)$ with standard deviation $\sigma$. Since the standard function arctan has the range $(-\pi / 2, \pi / 2)$, we use instead the function $\arctan 2(x, y)$, whose co-domain is $(-\pi, \pi]$. This function returns the angle between the vector (from origin to point $(x, y)$ ) and the positive direction of the $Y$-axis. Note that $\arctan 2(0,0)$ is undefined. Define the likelihood function as $\boldsymbol{L}=\boldsymbol{L}\left(\boldsymbol{P}_{B k}^{\prime}, \boldsymbol{P}_{A k}, \theta_{k} \mid R, T\right)$ and let $e_{k}=u_{k} \cos \alpha+v_{k} \sin \alpha+t_{1}-x_{k}^{\prime}$ and $g_{k}=v_{k} \cos \alpha-$ $u_{k} \sin \alpha+t_{2}-y_{k}^{\prime}$. We can then express $\boldsymbol{L}$ as

$$
\boldsymbol{L}=\frac{1}{(\sigma \sqrt{2 \pi})^{K}} \prod_{k=1}^{K} \exp \left[-\frac{\left(\widetilde{\theta}_{k}-\arctan 2\left(e_{k}, g_{k}\right)\right)^{2}}{2 \sigma^{2}}\right]
$$

which yields

$$
\ln \boldsymbol{L}=\sum_{k=1}^{K}\left[-\frac{\left(\widetilde{\theta}_{k}-\arctan 2\left(e_{k}, g_{k}\right)\right)^{2}}{2 \sigma^{2}}\right]-K \log (\sigma \sqrt{2 \pi})
$$

We thus obtain the cost function $f\left(\alpha, t_{1}, t_{2}\right)$, and the cost function is minimised for the values $\widetilde{\alpha}, \widetilde{t}_{1}, \widetilde{t}_{2}$ which satisfy

$$
\widetilde{\alpha}, \widetilde{t}_{1}, \widetilde{t}_{2}=\underset{\alpha, t_{1}, t_{2}}{\operatorname{argmin}} \sum_{k=1}^{K}\left(\widetilde{\theta}_{i}-\arctan 2\left(e_{k}, g_{k}\right)\right)^{2}
$$

The gradient of the cost function $f\left(\alpha, t_{1}, t_{2}\right)$ is evaluated as follows

$$
\begin{aligned}
\frac{\partial f}{\partial \alpha} & =\sum_{k=1}^{K} 2\left(\widetilde{\theta}_{k}-\arctan 2\left(e_{k}, g_{k}\right)\right) \frac{1}{e_{k}^{2}+g_{k}^{2}} \\
& \times\left(g_{k}\left(u_{k} \sin \alpha-v_{k} \cos \alpha\right)-e_{k}\left(v_{k} \sin \alpha+u_{k} \cos \alpha\right)\right) \\
\frac{\partial f}{\partial t_{1}} & =\sum_{k=1}^{K} 2\left(\widetilde{\theta}_{k}-\arctan 2\left(e_{k}, g_{k}\right)\right) \frac{-g_{k}}{e_{k}^{2}+g_{k}^{2}} \\
\frac{\partial f}{\partial t_{2}} & =\sum_{k=1}^{K} 2\left(\widetilde{\theta}_{k}-\arctan 2\left(e_{k}, g_{k}\right)\right) \frac{e_{k}}{e_{k}^{2}+g_{k}^{2}}
\end{aligned}
$$

A gradient descent MLE is initialised with the point $z_{1}=$ $\left[\begin{array}{lll}\widehat{\alpha} & \widehat{t}_{1} & \widehat{t}_{2}\end{array}\right]^{\top}$ obtained from $\boldsymbol{x}^{*}=\left[\widehat{t_{1}}, \widehat{t}_{2}, \cos \widehat{\alpha}, \sin \widehat{\alpha}\right]^{\top}$ in (12). In other words, the gradient descent MLE algorithm is initialised with the GTRS solution. With a sufficiently small fixed step size, we have convergence to a global minimum (experience shows that a fixed step size of 0.0001 virtually guarantees convergence to a global minimum).

\section{Simulations}

We conduct Monte Carlo simulations to investigate our proposed modifications to the algorithm in Section III to deal with the presence of noise. Table I records the position (meters) and bearing (degrees) data for the simulations executed in this section. The matrix $R$ and vector $T$ are given in the first row of Table II. The table data is noiseless, while in the simulations zero mean Gaussian noise of variance $\sigma^{2}$ is added to $\theta_{k}$. The quantities in the table are generated to closely reflect real-world UAV flight. Note that the maximum total number of measurements is 10 ; this is a reasonable number given the nature of the communication between Agent A and Agent B is likely to be RF, and the assumption that the measurements are completed in a sufficiently short time interval such that the INS does not drift.

In order to analyse the performance of the algorithms in Sections IV-A and IV-B, we define the error index

$$
E=\frac{\sum_{i=1}^{N} \sum_{k=1}^{K}\left\|\widehat{\boldsymbol{P}}_{B k}(i)-\boldsymbol{P}_{B k}\right\|}{N K} \quad \text { unit: meters }
$$

where $\widehat{\boldsymbol{P}}_{B k}(i)$ is the estimated value of $\boldsymbol{P}_{B k}$ at the $k^{\text {th }}$ time instant, for the $i^{t h}$ Monte Carlo iteration. Though not normalized, this can be viewed against the typical magnitudes of the distance between $\boldsymbol{P}_{A k}$ and $\boldsymbol{P}_{B k}$, which are of the order of hundreds of meters.

In the first set of simulations, we compare the performance of the GTRS algorithm with the performance of the gradient descent MLE (which is initialised by the GTRS solution). We let $K$ vary between 4 and 10 measurements, and set the noise statistic to $\sigma=3,9^{\circ}$. For each $K$ and $\sigma$, we execute $N=$ 100 Monte Carlo simulations and compute $E$ given in (20). The results for $\sigma=3^{\circ}$ and $\sigma=9^{\circ}$ are presented in Fig. 5 and 6 respectively. We observe that the gradient descent MLE (which is often considered a benchmark in performance) only yields minor performance gains when compared to the GTRS algorithm for both small and large $\sigma$.

In the second set of simulations, we investigate the gradient descent MLE at varying levels of noise. We let $K$ vary between 4 and 10 measurements. The noise standard deviation is set to $\sigma=3,6,9^{\circ}$. For each combination of $K$ and $\sigma$, we conduct $N=100$ Monte Carlo simulations. The error index $E$ is then computed. The results are displayed in Fig. 7. We conclude from both sets of simulations that taking more measurements gives better localisation accuracy. For $K \geq 6$, we see that performance is approximately proportional to $\sigma$. Below $K=6$, we observe from Fig. 7 that there is a collapse in the performance of the gradient descent MLE as noise increases from $\sigma=3^{\circ}$ to $\sigma=9^{\circ}$. We conjecture that this is because for $K<6$, at $\sigma=6,9^{\circ}$, the initialisation point obtained from the GTRS algorithm is well away from the attraction region of the global minimum of the MLE.

\section{CONCLUSIONS}

In this paper, we study the problem of UAV localisation in two-dimensional space under a GPS-denied environment using bearing measurements. By firstly considering noiseless measurements we show that generically, a unique localisation solution is obtained from four bearing measurements. Special cases are studied where a partial localisation solution is 
TABLE I

DATA USED For SimUlations

\begin{tabular}{|c|c|c|c|c|c|c|c|c|c|c|c|}
\hline \multirow{3}{*}{$\boldsymbol{P}_{B k}^{\prime}=$} & & $k=1$ & $k=2$ & $k=3$ & $k=4$ & $k=5$ & $k=6$ & $k=7$ & $k=8$ & $k=9$ & $k=10$ \\
\hline & $x_{k}^{\prime}$ & 1000 & {$[100]$} & {$[550]$} & {$[750]$} & 800 & 1200 & {$[1600]$} & {$[1700$} & {$[2100$} & {$[1900]$} \\
\hline & $y_{k}^{\prime}$ & -100 & 150 & 200 & 150 & -100 & -50 & 100 & 200 & 550 & 200 \\
\hline \multirow{2}{*}{$\boldsymbol{P}_{B k}=$} & $x_{k}$ & 1240 & 670 & {$[1060$} & 11190 & 1080 & 1430 & 1840 & 1980 & 2510 & 2140 \\
\hline & $y_{k}$ & {$[-380]$} & {$[360]$} & 130 & -30 & {$[-260]$} & {$[-460$} & -580 & -560 & -520 & -680 \\
\hline \multirow{2}{*}{$\boldsymbol{P}_{A k}=$} & {$\left[u_{k}\right.$} & {$[880]$} & {$[1090]$} & {$[1290]$} & {$[1550]$} & {$[1540]$} & {$[1860$} & 2240 & 2350 & {$[2280$} & $=2620^{\circ}$ \\
\hline & $v_{k}$ & {$[640]$} & 420 & 270 & {$[200]$} & {$[20$} & {$[-220]$} & -380 & -400 & {$[-660$} & -540 \\
\hline \multicolumn{2}{|l|}{$\theta_{k}$} & $-56.3^{\circ}$ & -45.0 & $-21.8^{\circ}$ & $-20.6^{\circ}$ & $-21.8^{\circ}$ & $-24.0^{\circ}$ & $-26.6^{\circ}$ & $-29.7^{\circ}$ & $-21.8^{\circ}$ & $-36.9^{\circ}$ \\
\hline
\end{tabular}

TABLE II

Two Solutions Obtained From Three Measurements

\begin{tabular}{|c|c|c|c|c|c|c|}
\hline & & $R$ & $T$ & $\boldsymbol{P}_{B 1}=\left[x_{1}, y_{1}\right]^{\top}$ & $\boldsymbol{P}_{B 2}=\left[x_{2}, y_{2}\right]^{\top}$ & $\boldsymbol{P}_{B 3}=\left[x_{3}, y_{3}\right]^{\top}$ \\
\hline Solution One, $\boldsymbol{P}_{B k}$ & & $\left.\begin{array}{c}-0.6 \\
0.8\end{array}\right]$ & {$[-220,-540]^{\top}$} & {$[1240,-380]^{\top}$} & {$[670,360]^{\top}$} & {$[1060,130]^{\top}$} \\
\hline Solution Two, $\boldsymbol{P}_{B k}{ }^{*}$ & {$\left[\begin{array}{c}0.715 \\
0.70\end{array}\right.$} & $\left.\begin{array}{c}-0.70 \\
0.715\end{array}\right]$ & {$[-59.5,-587.2]^{\top}$} & {$[1098.6,-393.3]^{\top}$} & {$[630.1,-415.45]^{\top}$} & {$[986.8,-136.2]^{\top}$} \\
\hline
\end{tabular}

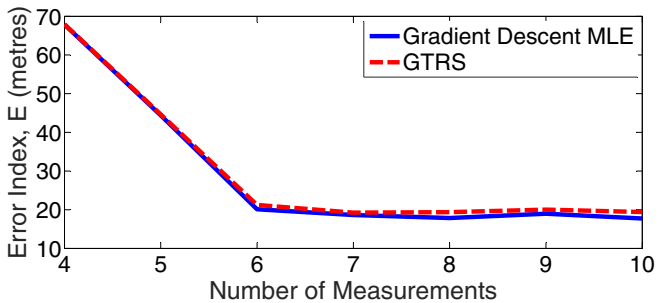

Fig. 5. Comparison of GTRS algorithm and gradient descent MLE, $\sigma=3^{\circ}$

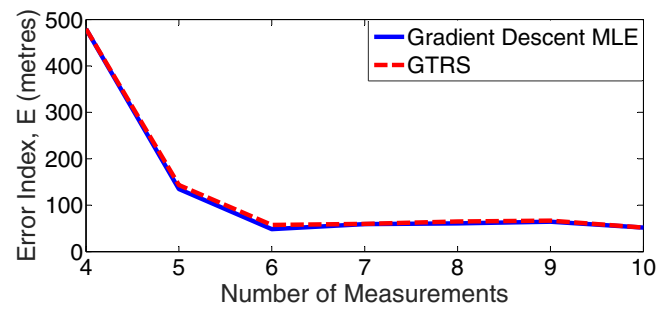

Fig. 6. Comparison of GTRS algorithm and gradient descent MLE, $\sigma=9^{\circ}$

obtained. Noise is then introduced into the bearing measurements and steps are made to minimise the effects of noise. We solve a GTRS to obtain an estimate of the unique localisation solution and this is used to initialise a maximum likelihood estimation. Numerical simulations are provided which show the effectiveness of the proposed localisation when there is bearing noise. Several directions exist for

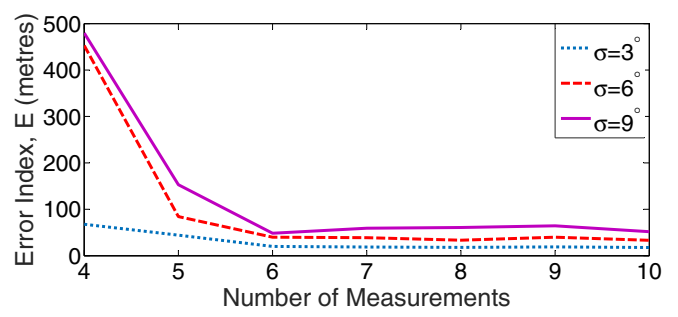

Fig. 7. Performance of the gradient descent MLE as noise increases. future work. An immediate extension is to consider two or more GPS-denied agents in a two-dimensional space. The two agent problem may be studied in three-dimensional space. Other future work may involve localisation using particle filtering or some form of extended Kalman filter.

\section{REFERENCES}

[1] B. D. O. Anderson, C. Yu, B. Fidan, and J. M. Hendrickx, "Rigid graph control architectures for autonomous formations," Control Systems, IEEE, vol. 28, no. 6, pp. 48-63, 2008.

[2] A. Bishop, B. D. O. Anderson, B. Fidan, P. Pathirana, and G. Mao, "Bearing-only localization using geometrically constrained optimization," Aerospace and Electronic Systems, IEEE Transactions on, vol. 45, no. 1, pp. 308-320, Jan 2009.

[3] Y. Oshman and P. Davidson, "Optimization of Observer Trajectories for Bearings-only Target Localization," IEEE Transactions on Aerospace and Electronic Systems, vol. 35, no. 3, pp. 892-902, 1999.

[4] K. Doğançay, "Bearings-only Target Localization Using Total Least Squares," Signal Processing, vol. 85, no. 9, pp. 1695-1710, 2005.

[5] R. M. Vaghefi, M. R. Gholami, and E. G. Ström, "Bearing-only Target Tocalization with Uncertainties in Observer Position," in IEEE 21 st International Symposium on Personal, Indoor and Mobile Radio Communications Workshops (PIMRC Workshops). IEEE, 2010, pp. 238-242.

[6] M. Ye, B. D. O. Anderson, and C. Yu, "Multiagent Self-Localization Using Bearing-only Measurements," in Proceedings of IEEE 52nd Annual Conference on Decision and Control (CDC). IEEE, 2013, pp. 2157-2162.

[7] B. Jiang, M. Deghat, and B. D. O. Anderson, "Translational Velocity Consensus Using Distance-only Measurements," in IEEE 52nd Annual Conference on Decision and Control, Dec 2013, pp. 2746-2751.

[8] S. Nardone, A. G. Lindgren, and K. F.Gong, "Fundamental Properties and Performance of Conventional Bearings-only Target Motion Analysis," IEEE Transactions on Automatic Control, vol. 29, no. 9, pp. 775-787, Sep 1984.

[9] T. Eren, W. Whiteley, and P. N. Belhumeur, "Using Angle of Arrival (Bearing) Information in Network Localization," in 45th IEEE Conference on Decision and Control. Citeseer, 2006.

[10] I. Shames, A. N. Bishop, and B. D. O. Anderson, "Analysis of Noisy Bearing-only Network Localization," IEEE Transactions on Automatic Control, vol. 58, no. 1, pp. 247-252, 2013.

[11] M. Deghat, B. D. O. Anderson, and Z. Lin, "Combined Flocking and Distance-based Shape Control of Multi-agent Formations," IEEE Transactions on Automatic Control, vol. 61, no. 7, pp. 1824-1837, July 2016.

[12] J. J. Moré, "Generalizations of the trust region problem," Optimization Methods and Software, vol. 2, no. 3-4, pp. 189-209, 1993.

[13] C. Forbes, M. Evans, N. Hastings, and B. Peacock, Statistical Distributions. John Wiley \& Sons, 2011. 